\title{
Topological Order in Gutzwiller Projected Wave Functions for Quantum Antiferromagnets
}

\author{
Tao $\mathrm{Li}$ \\ Department of Physics, Renmin University of China, Beijing, 100872, P.R.China \\ Hong-Yu Yang \\ Center for Advanced study, Tsinghua University ,Beijing 100084, China
}

(Dated: November 14, 2018)

\begin{abstract}
We study the topological order in RVB state derived from Gutzwiller projection of BCS-like mean field state. We propose to construct the topological excitation on the projected RVB state through Gutzwiller projection of mean field state with inserted $Z_{2}$ flux tube. We prove that all projected RVB states derived from bipartite effective theories, no matter the gauge structure in the mean field ansatz, are positive definite in the sense of the Marshall sign rule, which provides a universal origin for the absence of topological order in such RVB state.

PACS numbers: 75.10.Jm, 75.50.Ee, 75.40.Mg
\end{abstract}

It is widely believed that the strongly correlated systems may exhibit exotic ground state structures and support exotic excitations. The study of variational wave functions provide a unique way to uncover such exoticness. In the two dimensional quantum antiferromagnet(2DQAF), it is proposed that a featureless quantum spin liquid state called resonating valence bond(RVB) state may be realized[1]. The RVB state is a coherent superposition of spin singlet pairs and can be written as

$$
|\mathrm{RVB}\rangle=\sum_{\text {dimer covering }} a\left(i_{1} j_{1} ; \ldots ; i_{n} j_{n}\right) \prod_{k=1}^{n}\left(i_{k} j_{k}\right),
$$

in which $(i j)=\frac{1}{\sqrt{2}}(i \uparrow j \downarrow-i \downarrow j \uparrow)$ is a spin singlet pair(valence bond) between site $i$ and $j$. It is argued that the RVB state may support fractionalized excitation of $\operatorname{spin} \frac{1}{2}$ spinon [2, 3].

A systematic way to generate the RVB state is through Gutzwiller projection of BCS-like mean field state[1]. Such kind of projected RVB states are now widely used in the variational study of undoped and doped quantum antiferromagnet [4, 5, , 6, 7]. An important issue on the projected RVB state is to characterize its structure and understand its excitation in physical terms. This issue is addressed at the effective theory level by Wen who introduced the notion of quantum order [8]. However, an understanding at the wave function level is still lack and it is unclear to what extend is the prediction of effective theory applicable to projected wave functions. Fortunately, the projective method to generate the variational ground state also provides a systematic way to generate the low energy excitations. A variational wave function for low energy excitation is simply generated by Gutzwiller projection of mean field excited states [1]. Such a procedure has been followed by a number of authors to study quasiparticle excitations [6, 9, 10, 11, 12] and topological excitations 13, 14] on projected RVB wave functions.
In this paper, we use such a projective construction to study the topological excitation and topological order on the projected RVB wave functions.

The concept of topological order is the key notion to describe the structure of a RVB state and to understand the fractionalization of excitation on it $3,16,18,19]$. The topological order can be defined as the rigidity of a system against topological excitation(dubbed vison) in the bulk of the system. On a multiply connected manifold, the topological order manifests itself as the topological degeneracy of the ground state, in which case the ground state has a number of locally similar but globally distinct partners that differ by whether or not a vison threads each hole of the manifold [8, 13].

These ideas can be illustrated by the simple example of quantum dimer model [3, 16, 17]. In the quantum dimer model(QDM), the Hilbert space factorizes into even and odd topological sectors by the number of valence bonds that intersect a cut line which starts at the center of vison and ends at the boundary of the system(or infinity). A vison can be generated simply by reversing the sign of the amplitudes of odd sector dimer configurations [16]. The vison defined in this fashion behaves as a $\pi$ flux tube for an unpaired spin. Thus the topological order is intimately related to the coherent motion of fractionalized spin excitation in the RVB background.

At the Gaussian level, the projected RVB states are described by the slave Boson effective theories [8, 18, 20]. According to the slave Boson effective theory, a system with $Z_{2}$ gauge structure in the mean field ansatz has topological order and supports fractionalized excitations, while a $Z_{2}$ flux tube plays the role of the vison[19].

In their pioneering work, Ivanov and Senthil conjectured that a $Z_{2}$ gauge structure in the mean field ansatz is also essential for the corresponding projected RVB state to show topological order 13, 14, 15]. Based on the phenomenological $Z_{2}$ gauge theory of the underdoped cuprates [19], they proposed that a vison can be con- 
structed by projecting BCS state containing a superconducting vortex. They find numerically that RVB states derived from certain effective theory with $U(1)$ gauge structure, more specifically, the nearest-neighboring dwave RVB state(NND state), does not exhibit topological order. This is taken as evidence for their conjecture, since the vison - vortex analog is ill defined when the pairing term is gauged away in a $U(1)$ effective theory.

In this paper, we try to relate the topological order of projected RVB state to their phase structure in the Ising basis. We find the absence of topological order in the NND-type RVB states can be more naturally attributed to the Marshall sign rule of the wave function in the Ising basis, rather than the $U(1)$ gauge structure in the mean field ansatz.

The Marshall sign rule is a well known property of the antiferromagnetic Heisenberg model on bipartite lattice 22, 23]. According to the rule, the wave function of the ground state is real in the Ising basis and its sign is given by $(-1)^{N_{\downarrow}^{\text {even }}}$, in which $N_{\downarrow}^{\text {even }}$ is the number of down spins in the even sublattice. In this paper, we prove that all RVB states derived from bipartite slave Boson mean field states satisfy such a sign rule, no matter what is the gauge structure of the mean field ansatz. At the same time, we show a vison can be constructed by projecting a mean field state containing a $Z_{2}$ flux tube, rather than a superconducting vortex used by Ivanov and Senthil. Combining these two points allow us to show that RVB states derived from all bipartite mean field states, such as the NND state, do not support topological order. Thus the Marshall sign rule provides an universal origin for the absence of topological order for RVB state defined on bipartite lattices [25], while a Gaussian level effective theory fails for such a state.

In the slave Boson theory of RVB states [8, 18, 20], Fermionic slave particles $f_{i \alpha}$ are introduced to represent the $S U(2)$ spin variable as $\mathbf{S}_{i}=\frac{1}{2} \sum_{\alpha \beta} f_{i \alpha}^{\dagger} \sigma_{\alpha \beta} f_{i \beta}$. These slave particles are subjected to the constraint of $\sum_{\alpha} f_{i \alpha}^{\dagger} f_{i \alpha}=1$ to be a faithful representation of the spin algebra. At the saddle point level, a RVB state is given by a BCS-like mean field ansatz for the slave particles

$$
\mathrm{H}_{\mathrm{MF}}=\sum_{\langle i j\rangle}\left(\psi_{i}^{\dagger} U_{i j} \psi_{j}+\text { H.c. }\right)+\sum_{i} \lambda_{i}\left(f_{i \alpha}^{\dagger} f_{i \alpha}-1\right),
$$

in which $\psi_{i}=\left(\begin{array}{c}f_{i \uparrow} \\ f_{i \downarrow}^{\dagger}\end{array}\right)$. Here, $U_{i j}=\left(\begin{array}{cc}-\chi_{i j}^{*} & \Delta_{i j} \\ \Delta_{i j}^{*} & \chi_{i j}\end{array}\right)$ denote the mean field RVB order parameters, while $\lambda_{i}$ are the Lagrangian multipliers introduced to enforce the mean field constraint. The fluctuation of the RVB order parameters are treated by effective gauge theories [18]. When the gauge symmetry of the mean field ansatz is broken to $Z_{2}$, the effective theory is a $Z_{2}$ gauge theory [18, 19]. Since the $Z_{2}$ gauge fluctuation is gapped at the Gaussian level, it is believed that a $Z_{2}$ effective theory describes a phase with truly fractionalized excitations.

The ground state of the mean field Hamiltonian has the form of Cooper pair condensate 21],

$$
|\Psi\rangle=\exp \left(\sum_{i j} a_{i j}\left(f_{i \uparrow}^{\dagger} f_{j \downarrow}^{\dagger}-f_{i \downarrow}^{\dagger} f_{j \uparrow}^{\dagger}\right)\right)|0\rangle
$$

in which $a_{i j}$ is the wave function of a Cooper in real space. The RVB state is given by Gutzwiller projection of the mean field ground state,

$$
|\mathrm{RVB}\rangle=\mathrm{P}_{\mathrm{G}}|\Psi\rangle=\mathrm{P}_{\mathrm{G}}\left(\sum_{i j} a_{i j}\left(f_{i \uparrow}^{\dagger} f_{j \downarrow}^{\dagger}-f_{i \downarrow}^{\dagger} f_{j \uparrow}^{\dagger}\right)\right)^{\frac{N}{2}}|0\rangle,
$$

in which $\mathrm{P}_{\mathrm{G}}=\prod_{i}\left(1-n_{i \uparrow} n_{i \downarrow}\right)$ and $N$ is the number of lattice site.

The topological order can be checked by examining on a tours the orthogonality of the RVB states that differs in the number of trapped visons in the holes of the torus. Here, we propose a more transparent way to construct the vison wave function. In the effective theory context, a vison is nothing but a $Z_{2}$ gauge flux tube. Hence, we propose to construct the vison wave function through Gutzwiller projection of mean field state with an inserted $Z_{2}$ gauge flux tube. The only effect of the inserted $Z_{2}$ flux tube is to reverse the sign of mean field RVB order parameters $\chi_{i j}$ and $\Delta_{i j}$ on bonds that intersect the cut line defining the vison, which amounts to changing the boundary condition across the cut line from periodic to anti-periodic(or vice versa). In Ivanov and Senthil's work, the role of a trapped superconducting vortex is also to change the boundary condition across the cut line. Thus our construction is equivalent to theirs but is applicable in more general situations.

Now we show that the absence of topological order in the NND-type RVB state can be attributed the Marshall sign rule. The NND theory belongs to the general class of $U(1)$ bipartite effective theory, which contains a $U(1)$ gauge structure in the mean field ansatz and is bipartite in the sense that $\chi_{i j}$ and $\Delta_{i j}$ only connect sites in opposite sublattices. Below we show that the RVB states derived from such theories satisfy the Marshall sign rule. Since a $U(1)$ bipartite theory is still $U(1)$ bipartite with the insertion of a $Z_{2}$ flux tube, the RVB state with a trapped vison also satisfy the Marshall sign rule and will not be orthogonal to the RVB state with no trapped vison in general case 25]. Thus RVB states in this class are unlikely to exhibit topological order.

For convenience's sake, we work in the gauge with only hopping term,

$$
\mathrm{H}_{\mathrm{MF}}=\sum_{\langle i j\rangle, \sigma}\left(\chi_{i j} f_{i \sigma}^{\dagger} f_{j \sigma}+\text { H.c. }\right) \text {. }
$$


The mean field ground state of $\mathrm{H}_{\mathrm{MF}}$ is given by

$$
|\Psi\rangle=\prod_{\xi_{m}<0} f_{m \uparrow}^{\dagger} f_{m \downarrow}^{\dagger}|0\rangle
$$

in which $f_{m}^{\dagger}=\sum_{i} \varphi_{m}(i) f_{i}^{\dagger}$ denotes the eigenstate of $\mathrm{H}_{\mathrm{MF}}$ of eigenvalue $\xi_{m}$. For a bipartite Hamiltonian, the eigenvalues appear in plus-minus pairs. The corresponding eigenfunctions are

$$
\begin{gathered}
\varphi_{m}(i)=\varphi_{m}^{e}(i)+\varphi_{m}^{o}(i) \\
\varphi_{\bar{m}}(i)=\varphi_{m}^{e}(i)-\varphi_{m}^{o}(i),
\end{gathered}
$$

in which $\varphi_{m}^{e}(i)$ and $\varphi_{m}^{o}(i)$ are the components of the eigenfunctions in the subspace of even and odd sublattices. From the orthonormality of $\varphi_{m}(i)$, it is easy to show that $\varphi_{m}^{e}(i)$ and $\varphi_{m}^{o}(i)$ form complete and orthonormal basis in their respective subspaces. Thus, the eigenvectors with $\xi_{m}<0$ suffice to expand the subspaces of each sublattices. This property of the bipartite Hamiltonian is the key to establish the Marshall sign rule in the projected RVB states.

In the basis expanded by $\prod_{k=1, \frac{N}{2}} f_{i_{k} \uparrow}^{\dagger} f_{j_{k} \downarrow}^{\dagger}|0\rangle$, the amplitude of the above RVB state is given by the following Slater determinant

$$
\psi=\left|\begin{array}{ccc}
\varphi_{1}\left(i_{1}\right) & \cdots & \varphi_{1}\left(i_{\frac{N}{2}}\right) \\
\vdots & & \vdots \\
\varphi_{\frac{N}{2}}\left(i_{1}\right) & \cdots & \varphi_{\frac{N}{2}}\left(i_{\frac{N}{2}}\right)
\end{array}\right|\left|\begin{array}{ccc}
\varphi_{1}\left(j_{1}\right) & \cdots & \varphi_{1}\left(j_{\frac{N}{2}}\right) \\
\vdots & & \vdots \\
\varphi_{\frac{N}{2}}\left(j_{1}\right) & \cdots & \varphi_{\frac{N}{2}}\left(j_{\frac{N}{2}}\right)
\end{array}\right| .
$$

For convenience's sake, we define a reference spin configuration in which all up(down) spins sit in the even(odd) sublattice. The amplitude of this spin configuration is given by

$\psi_{\text {ref }}=\left|\begin{array}{ccc}\varphi_{1}^{e}\left(i_{1}\right) & \cdots & \varphi_{1}^{e}\left(i_{\frac{N}{2}}\right) \\ \vdots & & \vdots \\ \varphi_{\frac{N}{2}}^{e}\left(i_{1}\right) & \cdots & \varphi_{\frac{N}{2}}^{e}\left(i_{\frac{N}{2}}\right)\end{array}\right|\left|\begin{array}{ccc}\varphi_{1}^{o}\left(j_{1}\right) & \cdots & \varphi_{1}^{o}\left(j_{\frac{N}{2}}\right) \\ \vdots & & \vdots \\ \varphi_{\frac{N}{2}}^{o}\left(j_{1}\right) & \cdots & \varphi_{\frac{N}{2}}^{o}\left(j_{\frac{N}{2}}\right)\end{array}\right|$.

Since the row vector $\left(\varphi_{m}^{e}\left(i_{1}\right) \cdots \varphi_{m}^{e}\left(i_{\frac{N}{2}}\right)\right)$ form complete and orthonormal basis in the subspace of even sublattice, the column vector $\left(\varphi_{1}^{e}(i) \cdots \varphi_{\frac{N}{2}}^{e}(i)\right)^{T}$ also form a complete and orthonormal basis. The same is true for the odd sublattice.

Now consider a general spin configurations. With no loss of generality, we consider the spin configuration derived from the reference configuration through exchange of the first $k$ up spins and the first $k$ down spins. One easily verifies that the inner product of the amplitudes for thess two configurations, $\psi_{k}^{*} \psi_{r e f}$, is given by the square modulus of a $k$-th order determinant

$$
\psi_{k}^{*} \psi_{\text {ref }}=|| \begin{array}{ccc}
s\left(j_{1} i_{1}\right) & \cdots & s\left(j_{1} i_{k}\right) \\
\vdots & & \vdots \\
s\left(j_{k} i_{1}\right) & \cdots & s\left(j_{k} i_{k}\right)
\end{array}||^{2}
$$

in which $s\left(j_{l}, i_{n}\right)=\sum_{m=1, \frac{N}{2}} \varphi_{m}^{o *}\left(j_{l}\right) \varphi_{m}^{e}\left(i_{n}\right), i_{n}$ and $j_{l}$ denote the coordinates of the exchanged $k$ up spins and $k$ down spins.

Thus, the amplitude of the RVB state derived from Eq.(5) is positive definite up to a global phase. Taking into account the sign change due to Fermion exchange, we arrive at the conclusion that the projected RVB states derived from $U(1)$ bipartite theories satisfy the Marshall sign rule and thus are very unlikely to exhibit topological order. However, it is not clear whether the $U(1)$ gauge structure or the bipartite nature of the theory is responsible for the absence of topological order. To clarify this point, we examine the phase structure of projected RVB state derived from an arbitrary bipartite effective theory.

The mean field ansatz for an arbitrary bipartite theory is given by

$$
\begin{aligned}
\mathrm{H}_{\mathrm{MF}}= & \sum_{\langle i j\rangle, \sigma}\left(\chi_{i j} f_{i \sigma}^{\dagger} f_{j \sigma}+H . C .\right) \\
& +\sum_{\langle i j\rangle}\left(\Delta_{i j}\left(f_{i \uparrow}^{\dagger} f_{j \downarrow}^{\dagger}+f_{j \uparrow}^{\dagger} f_{i \downarrow}^{\dagger}\right)+H . C .\right),
\end{aligned}
$$

in which $\chi_{i j}$ and $\Delta_{i j}$ connect sites on different sublattices and are otherwise arbitrary(note other details of the lattice, such as its dimension, spatial symmetry and topology, are all irrelevant for our discussion). The Lagrangian multipliers are set to zero since the theory is particle-hole symmetric. To facilitate the proof, we make a particle-hole transformation on down spins [4],

$$
f_{i \downarrow} \longrightarrow \epsilon_{i} \tilde{f}_{i \downarrow}^{\dagger}
$$

in which $\epsilon_{i}=1$ for $i \in$ even sublattice and $\epsilon_{i}=-1$ for $i \in$ odd sublattice. The transformed Hamiltonian reads

$$
\begin{aligned}
\tilde{\mathrm{H}}_{\mathrm{MF}}= & \sum_{\langle i j\rangle}\left(\chi_{i j} f_{i \uparrow}^{\dagger} f_{j \uparrow}+H . c .\right)+\sum_{\langle i j\rangle}\left(\chi_{i j}^{*} \tilde{f}_{i \downarrow}^{\dagger} \tilde{f}_{j \downarrow}+H . c .\right) \\
& +\sum_{\langle i j\rangle}\left(\epsilon_{j} \Delta_{i j}\left(f_{i \uparrow}^{\dagger} \tilde{f}_{j \downarrow}-f_{j \uparrow}^{\dagger} \tilde{f}_{i \downarrow}\right)+H . c .\right) .
\end{aligned}
$$

The eigenvector of this Hamiltonian is given by $\gamma_{n}^{\dagger}=$ $\sum_{i}\left(u_{n}(i) f_{i \uparrow}^{\dagger}+v_{n}(i) \tilde{f}_{i \downarrow}^{\dagger}\right)$. Since the Hamiltonian is bipartite, its eigenvalues appear in plus-minus pairs. The corresponding eigenvectors are given by

$$
\left(\begin{array}{l}
u_{n}(i) \\
v_{n}(i)
\end{array}\right)^{\text {even }} \pm\left(\begin{array}{c}
u_{n}(i) \\
v_{n}(i)
\end{array}\right)^{\text {odd }},
$$

in which $\left(\begin{array}{l}u_{n}(i) \\ v_{n}(i)\end{array}\right)^{\text {even }}$ and $\left(\begin{array}{c}u_{n}(i) \\ v_{n}(i)\end{array}\right)^{\text {odd }}$ are the projections of the eigenvectors in the even and odd sublattices. For a bipartite Hamiltonian, these sublattice projections form complete and orthonormal basis in their respective subspaces. At the same time, since the system is spin 
rotational symmetric, the eigenvalues are two fold degenerate. Specifically, if $\left(u_{m}(i), v_{m}(i)\right)$ is an eigenvector of the Hamiltonian, then $\left(v_{m}^{*}(i),-u_{m}^{*}(i)\right)$ is a eigenvector of the Hamiltonian with the same eigenvalue. Thus, the mean field ground state of the Hamiltonian can be written as

$$
\begin{aligned}
|\Psi\rangle=\prod_{\xi_{m}<0} \gamma_{m}^{\dagger}|\tilde{0}\rangle & =\prod_{m=1}^{\frac{N^{\prime}}{2}}\left(u_{m}(i) f_{i \uparrow}^{\dagger}+v_{m}(i) \tilde{f}_{i \downarrow}^{\dagger}\right) \\
& \times\left(v_{m}^{*}(i) f_{i \uparrow}^{\dagger}-u_{m}^{*}(i) \tilde{f}_{i \downarrow}^{\dagger}\right)|\tilde{0}\rangle,
\end{aligned}
$$

in which $\zeta_{m}$ denotes the eigenvalue, and $|\tilde{0}\rangle$ denotes the vacuum of $f_{i \uparrow}$ and $\tilde{f}_{i \downarrow}$. The prime on the product indicates that the product is taken over the eigenvectors with negative eigenvalues.

We now project the wave function into the subspace of no double occupancy. At half filling, the up spins and the holes of the down spins should occupy the same set of lattice sites in the projected wave function. Thus a general spin configuration can be expressed in the Fock basis as $\prod_{m=1, \frac{N}{2}} f_{i_{m} \uparrow}^{\dagger} \prod_{m=1, \frac{N}{2}} \tilde{f}_{i_{m} \downarrow}^{\dagger}|\tilde{0}\rangle$. The amplitude of the projected RVB state for this configuration is given by the following Slater determinant

$$
\psi=\left|\begin{array}{cccccc}
u_{1}\left(i_{1}\right) & \cdots & u_{1}\left(i_{\frac{N}{2}}\right) & v_{1}\left(i_{1}\right) & \cdots & v_{1}\left(i_{\frac{N}{2}}\right) \\
\vdots & & \vdots & \vdots & & \vdots \\
u_{\frac{N}{2}}\left(i_{1}\right) & \cdots & u_{\frac{N}{2}}\left(i_{\frac{N}{2}}\right) & v_{\frac{N}{2}}\left(i_{1}\right) & \cdots & v_{\frac{N}{2}}\left(i_{\frac{N}{2}}\right) \\
v_{1}^{*}\left(i_{1}\right) & \cdots & v_{1}^{*}\left(i_{\frac{N}{2}}\right) & -u_{1}^{*}\left(i_{1}\right) & \cdots & -u_{1}^{*}\left(i_{\frac{N}{2}}\right) \\
\vdots & & \vdots & \vdots & & \vdots \\
v_{\frac{N}{2}}^{*}\left(i_{1}\right) & \cdots & v_{\frac{N}{2}}^{*}\left(i_{\frac{N}{2}}\right) & -u_{\frac{N}{2}}^{*}\left(i_{1}\right) & \cdots & -u_{\frac{N}{2}}^{*}\left(i_{\frac{N}{2}}\right)
\end{array}\right| .
$$

Following essentially the same steps that we have detailed in the $U(1)$ case, one can show that the column vectors, $\left(u_{1}\left(i_{e}\right) \cdots u_{\frac{N}{2}}\left(i_{e}\right), v_{1}^{*}\left(i_{e}\right) \cdots v_{\frac{N}{2}}^{*}\left(i_{e}\right)\right)^{T}$ and $\left(v_{1}\left(i_{e}\right) \cdots v_{\frac{N}{2}}\left(i_{e}\right),-u_{1}^{*}\left(i_{e}\right) \cdots-u_{\frac{N}{2}}^{*}\left(i_{e}\right)\right)^{T}$ for $i_{e} \in$ even sublattice, and $\left(u_{1}\left(i_{o}\right) \cdots u_{\frac{N}{2}}\left(i_{o}\right), v_{1}^{*}\left(i_{o}\right) \cdots v_{\frac{N}{2}}^{*}\left(i_{o}\right)\right)^{T}$ and $\left(v_{1}\left(i_{o}\right) \cdots v_{\frac{N}{2}}\left(i_{o}\right),-u_{1}^{*}\left(i_{o}\right) \cdots-u_{\frac{N}{2}}^{*}\left(i_{o}\right)\right)^{T}$ for $i_{o} \in$ odd sublattice, form complete and orthonormal basis in their respective subspaces. From this orthogonality, one can show that $\psi_{k}^{*} \psi_{\text {ref }}$ is still given by Eq.(7), with the matrix element now given by $s\left(j_{l}, i_{n}\right)=$ $\sum_{m=1, \frac{N}{2}}\left(u_{m}^{*}\left(j_{l}\right) u_{m}\left(i_{n}\right)+v_{m}^{*}\left(j_{l}\right) v_{m}^{*}\left(i_{n}\right)\right)$. As before, $\psi_{\text {ref }}$ denotes the amplitude of the reference spin configuration with all up spins occupying the even sublattice and all down spins occupying the odd sublattice, while $\psi_{k}$ denotes the amplitude of a general spin configuration with $k$ up spins occupying the odd sublattice. $i_{n}$ and $j_{l}$ denote the coordinates of the exchanged $k$ up spins and $k$ down spins.

Thus, the amplitude of the RVB state derived from Eq.(8) in the basis expanded by $\prod_{k=1, \frac{N}{2}} f_{i_{k} \uparrow}^{\dagger} \tilde{f}_{i_{k} \downarrow}^{\dagger}|\tilde{0}\rangle$ is also positive definite up to a global phase. It is easy to check that the Marshall sign rule has been built into this basis. Thus projected RVB state derived from an arbitrary bipartite effective theory satisfies the Marshall sign rule and are very unlikely to show topological order, no matter the gauge structure of the mean field ansatz. Since the topological order is directly responsible for the existence of fractionalized excitations, our result also implies that the Marshall sign rule provides a universal origin of confining force for fractionalized excitations on bipartite lattice.

Our proof of the Marshall sign rule makes it clear that a $Z_{2}$ gauge structure in the mean field ansatz alone is not sufficient for the derived RVB state to show topological order. To illustrate this point, we have checked the topological order in the RVB state derived from a $Z_{2}$ bipartite theory with the following mean field ansatz on square lattice

$$
\begin{array}{r}
U_{i, i+x}=-\tau_{3}+\tau_{1} ; U_{i, i+y}=-\tau_{3}-\tau_{1} \\
U_{i, i+3 x}==-\tau_{3}+\tau_{2} ; U_{i, i+3 y}=-\tau_{3}-\tau_{2},
\end{array}
$$

in which $\tau_{1}, \tau_{2}$ and $\tau_{3}$ denote the three Pauli matrices. In Figure 1a, we plot the overlap between the state with periodic-antiperiodic boundary condition and that with antiperiodic-periodic boundary condition on a torus. The result shows clearly the absence of topological degeneracy in such an RVB state. On the other hand, since the definition of vison is now independent of the gauge structure of the effective theory, neither is a $Z_{2}$ gauge structure in the mean field ansatz necessary for the projected RVB state to show topological order. To illustrate this point, we have checked the topological degeneracy in the RVB state derived from a $U(1)$ nonbipartite theory with the following mean field ansatz

$$
\begin{array}{r}
U_{i, i+x}=-\tau_{3} ; U_{i, i+y}=-(-1)^{i_{x}} \tau_{3} ; \\
U_{i, i+x+y}=-3(-1)^{i_{x}} \tau_{3} .
\end{array}
$$

This ansatz describes a $U(1)$ RVB state on anisotropic triangular lattice. The isotropic version of this ansatz is found to be a good approximation for the ground state of the Heisenberg model on triangular lattice 24]. The overlap for this RVB state is shown in Figure 1b. The result indicates that the RVB state derived from such a $U(1)$ theory exhibits topological degeneracy. Thus the gauge structure in the mean field ansatz seems to be not a good teller for existence of the topological order in the projected RVB state.

In summary, we have proposed a new way to construct topological excitation on projected RVB states. We find the Marshall sign rule provides a universal origin for the absence of topological order for RVB states derived from bipartite effective theories. This indicates that a Gaussian level effective theory is insufficient for RVB states defined on bipartite lattices. 


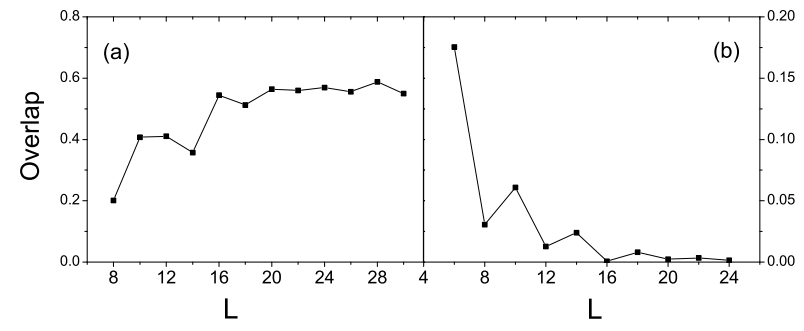

FIG. 1: Overlaps between the RVB state with periodicantiperiodic boundary condition and the RVB state with antiperiodic-periodic boundary condition on a torus of size L.(a) $Z_{2}$ bipartite case, (b) $U(1)$ non-bipartite case.

This work is supported by NSFC Grant No.90303009. Discussions with Z. Y. Weng, X. Q. Wang, L. Yu, M. Ogata and A. Paramekanti are acknowledged.

[1] P. W. Anderson Science 235, 1196 (1987).

[2] Z. Zou and P.W. Anderson, Phys. Rev. B 37, 627 (1988).

[3] S. Kivelson, D.Rokhsar and J. Sethna, Phys. Rev. B 35, 8865 (1987).

[4] H. Yokoyama and H. Shiba, J. Phys. Soc. Jpn. 57, 2482 (1988).

[5] C. Gros, Phys. Rev. B 38, 931 (1988).

[6] T.K. Lee C. M. Ho and N. Nagaosa, Phys. Rev. Lett. 90, 067001 (2003).

[7] S. Yunoki and S. Sorella, Phys. Rev. Lett. 92, 157003 (2004).
[8] X. G. Wen, Phys. Rev. B 65, 165113 (2002).

[9] S. Yunoki E. Dagotto and S. Sorella, Phys. Rev. Lett. 94, 037001 (2005).

[10] S. Yunoki, Phys. Rev. B 72, 092505 (2005).

[11] N. Fukushima, B. Edegger, V. N. Muthukumar and C. Gros, Phys. Rev. B, 72, 144505 (2005).

[12] A. Paramekanti M. Randeria and N. Trivedi, Phys. Rev. B 70, 054504 (2004).

[13] D. A. Ivanov and T. Senthil, Phys. Rev. B 66, 115111 (2002).

[14] A. Paramekanti, M. Randeria, and N. Trivedi, Phys. Rev. B 71, 094421 (2005).

[15] S. Sorella, L. Capriotti, F. Becca, and A. Parola, Phys. Rev. Lett. 91, 257005 (2003).

[16] N. Read and B. Chakraborty, Phys. Rev. B 40, 7133 (1989).

[17] A. Ralko, M. Ferrero, F. Becca, D. Ivanov and F. Mila Phys. Rev. B 71, 224109 (2005).

[18] X. G. Wen, Phys. Rev. B 44, (2664) (1991).

[19] T. Senthil and Matthew P. A. Fisher, Phys. Rev. B 63, 134521 (2001).

[20] P.A. Lee, N. Nagaosa, T.K. Ng, and X.G. Wen, Phys. Rev. B 57, 6003 (1998).

[21] A. Himeda, T. Kato and M. Ogata, Phys. Rev. Lett. 88 117001 (2002).

[22] W. Marshall, Proc. R. Soc. London A 23248 (1955).

[23] Z.Y. Weng, D.N. Sheng, Y.C. Chen and C.S. Ting, Phys. Rev. B 55, 3894 (1997).

[24] S. Yunoki and S. Sorella, Phys. Rev. B 74,014408 (2006).

[25] Our proof does not exclude the possibility that two positive definite wave function be orthogonal in the thermodynamic limit. Thus, the Marshall sign rule only provides a possible origin for the absence of the topological order and is insufficient to guarantee the absence of the topological order. 